DOI 10.37882/2500-3682.2020.10.08

\title{
ОБЗОР СОВРЕМЕННЫХ ПСИХОЛОГИЧЕСКИХ ПРОГРАММ ПРЕОДОЛЕНИЯ АКАДЕМИЧЕСКОЙ ПРОКРАСТИНАЦИИ СТУДЕНТОВ В УСЛОВИЯХ ОБУЧЕНИЯ В ВУЗЕ. РОЛЬ ПРЕПОДАВАТЕЛЕЙ В ПСИХОКОРРЕКИЦОННОМ ПРОЦЕССЕ
}

\section{REVIEW OF MODERN PSYCHOLOGICAL PROGRAMS FOR OVERCOMING ACADEMIC PROCRASTINATION OF STUDENTS IN A UNIVERSITY STUDY ENVIRONMENT. THE ROLE OF TEACHERS IN THE PSYCHOCORRECTIONAL PROCESS}

\section{A. Konovalova}

Summary: All people, to one degree or another, tend to postpone things for later, but part of the population in the scientific literature is called «chronic procrastinators», which are characterized by constant postponement of significant tasks in everyday, professional and academic activities [16, p178]. At the heart of procrastination is a complex combination of biological, psychological and sociocultural factors that are not fully explored, despite the abundance of scientific publications [37, p.1179; 41, p. 1415]. The most common form of procrastination is academic procrastination, common among schoolchildren and students. Sabotage of learning harms not only the quality of education, but also affects the mental health of young people, the quality of their future professional activities, as well as indirectly affects the satisfaction with the work of the teaching staff.

Keywords: procrastination, academic procrastination, the role of the teacher in reducing student procrastination, psychocorrection of procrastination.
Коновалова Анастасия Вадимовна

старший преподаватель, Российский Государственный Гуманитарный Университет konanavad@gmail.com

Аннотация: Всем людям в той или иной степени свойственно откладывать дела на потом, но часть популяции в научной литературе называют «хроническими прокрастинаторами», для которых характерно постоянное откладывание значимых задач в бытовой, профессиональной и академической деятельности [16,p178]. В основе прокрастинации лежит сложная комбинация биологических, психологических и социокультурных факторов, которые не до конца исследованы, не смотря на обилие научных публикаций [37,р.1179; 41, p.1415]. Наиболее распространённой формой откладывания является академическая прокрастинация, свойственная школьникам и студентам. Саботаж обучения вредит не только качеству образования, но также влияет на психическое здоровье молодых людей, на качество их будущей профессиональной деятельности, а также опосредованно касается удовлетворенности работой профессорско-преподавательского состава.

Ключевые слова: прокрастинация, академическая прокрастинация, роль преподавателя в снижении прокрастинации студентов, психокоррекция прокрастинации. билие исследований, посвященных поиску факторов формирования и поддержания прокрастинации, не дают пока полного представления о ее природе [76,p.28]. Очевидным является то, что прокрастинаторы испытывают дистресс [8,p.228] и хотели бы преодолеть откладывание [34,р.131]. Не смотря на пугающую распространённость и негативное влияние откладывания на качество жизни людей (по данным Klingsieck, Grund, Schmid, \& Fries около 70\% современных студентов являются хроническими прокрастинаторами), на сегодняшний день нет четких научно обоснованных протоколов преодоления прокрастинации, которые могли бы эффективно применяться практикующими психологами, нет рекомендаций для учителей, преподавателей вузов и тьюторов [37, р.1180]. В большом количестве фундаментальных исследований описываются разного рода негативные последствия прокрастинации [13,p.252], однако работ, посвященных внедрению и анализу эффективности психокоррекционных программ, направленных на преодоление откладывания, за рубежом единицы [9,p.400] В России таких исследований на данный момент нет совсем.

\section{Акацемическая прокрастинация}

В большинстве публикаций прокрастинация рассматривается как устойчивая черта личности или как стилевая характеристика деятельности. Ряд авторов предполагает, что академическая прокрастинация чаще встречается среди студентов младших курсов и уменьшается к окончанию обучения [15, р.82]. Можно предположить, что данная закономерность объясняется тем, 
что молодые люди в начале обучения обладают менее развитыми навыками саморегуляции, переживают адаптацию к новым условиям обучения, не имеют достаточных навыков планирования своего времени. Возможно, по мере взросления у студентов есть шанс преодолеть откладывание, и прокрастинация не превратится в образ жизни и не генерализируется с обучения на другие виды деятельности [6,р.395].

Вероятно, высокий показатель прокрастинации на младших курсах связан с удлинением периода детства у современного поколения, а, соответственно, с наличием таких психологических характеристик детства, как незрелость эмоционально-волевой сферы, неустойчивость смысложизненных ориентиров и т.д. Во многих исследованиях последних лет период старшего подростничества определяется возрастными рамками от 14 до 21 года, период юности - до 30 лет [1, с. 542]. Очевидно, что программы психолого-педагогической помощи должны быть направленны именно на эту аудиторию. Стоит упомянуть несколько изначальных сложностей в реализации подобного начинания. Во-первых, студенты, как правило, не идентифицирует прокрастинацию как проблему. Во-вторых, даже осознав трудность молодые люди, финансово зависимые от родителей, не имеют возможности обращаться к частнопрактикующим психологам, а государственные психологические службы есть не во всех ВУЗах. В-третьих, профессорско-преподавательский состав, как правило, не знаком с проблемой прокрастинации, не ориентирован на помощь студентам, а предпочитает скорее транслировать знания. И, наконец, самое важное - на данный момент нет проверенной и простой программы преодоления академической прокрастинации.

Академическая прокрастинация - явление системное, она не просто проблема конкретного студента. Откладывание или саботаж учебных задач студентом приводит к изменению стиля учебной деятельности его одногруппников, влияет на межличностное общение и психологический климат в коллективе, на эмоциональную сферу преподавателей. В последние годы в научной литературе прокрастинация все чаще понимается как проблема саморегуляции [28,р.207]. Саморегуляция имеет мощный биологический фундамент, в социальном плане требует формирования определенных навыков, которым молодых людей нигде целенаправленно не обучают [44,p.68]. При этом стратегии саморегуляции достаточно разнообразны, к ним можно отнести когнитивные, метакогнитивные, эмоциональные и поведенческие. Перед субъектом стоит задача осознанно использовать некоторый набор стратегий для повышения эффективности своей деятельности. Студенты, по всей видимости, с этой задачей не справляются как в силу подросткового возраста, так и из-за отсутствия необходимых знаний, подготовки, внешней поддержки [27,р.388]. Как правило, в колледжах и вузах поддержка студенчества отсутствует совсем или невелика, и ребята остаются с проблемой саморегуляции один на один [43,p.109].

Интересно, что частота проявлений академической прокрастинации не зависит от культуры, пола, гендера, национальности [16,p.920; 24,p.37]. Академическая прокрастинация касается всех форм учебной деятельности: подготовки к семинарским занятиям, экзаменам, написанию эссе и курсовых работ, в результате выливается в низкую успеваемость, академическую задолженность [22,p.47; 1,p.8; 7,p.202; 14,p.121].

В самом масштабном на данный момент аналитическом обзоре исследований прокрастинации отмечается, что прокрастинация входит в состав такой личностной черты как добросовестность и отрицательно взаимосвязана с психологическим благополучием, осмысленностью жизни. Коррелятами прокрастинации выступают импульсивность, особенности мотивационной сферы и ряд характеристик самих учебных задач [10,p.407]. Основываясь на теории временной мотивации, Steel [35,p.65;36,p.78] предположил, что люди будут откладывать выполнение задачи, если выполняется хотя бы одно из следующих условий:

- субъект не верит в успешность решения задачи;

- предполагается, что процесс решения будет неприятным, а результат не будет представлять ценности;

- присутствует длинный временной интервал между выполнением задачи и получением какой-либо значимой выгоды от ее решения (награды).

Исследования, проведенные в русле когнитивноповеденческого направления, обнаружили многочисленные взаимосвязи академической прокрастинации с внутренними установками. Так для прокрастинаторов характерны большее количество негативных автоматических мыслей [8,p.228], низкая мотивация достижений, негативные мысли о себе, низкая самооценка [35,p.47], снижение концентрации внимания [34,р.242]страх неудачи [13,p.257], низкие способности планирования деятельности [12,p1173], узкий репертуар адаптивных реакций [30,p.288] снижение психофизиологического ресурса [10,p.411] и нарушение исполнительных функций [29,p.352]. В других исследованиях подчеркивались негативные последствия академической прокрастинации: неблагоприятное поведение в отношении собственного здоровья, ухудшение самочувствия, низкая успеваемость, добровольная социальная изоляция, избегание близких отношений [15,p.31; 20,p.63; 35,p.240; 38,p.57].

Шроу, Уодкинс и Олафсон [33,р.12] провели масштабное исследование прокрастинации с целью выяснения ее связи с успеваемостью. Результаты показали, что ака- 
демическая прокрастинация в некоторых случаях может иметь адаптивный аспект. В самоотчетах части студентов прокрастинация описывалась как осознанное решение отложить деятельность для того, чтобы сэкономить ресурсы, более грамотно распределить время, повысить эффективность деятельности на финишной прямой. Эти студенты не описывали негативные последствия прокрастинации ни в форме субъективно переживаемого дистресса, ни в виде снижения академической успеваемости. Авторы также обнаружили, что студенты иногда прокрастинировали намеренно, чтобы улучшить общее качество жизни. Они достаточно спокойно себя чувствовали в течение семестра, могли позволить себе свободное время, любимые занятия, а в конце семестра испытывали рост напряжения на протяжение двух-трех недель. Можно предположить, что эти студенты использовали эмоциональное напряжение для повышения своей мотивации и эффективности решения субъективно неприятных задач. Данные выводы согласуются с другими исследованиями, авторы которых предполагали, что академический прокрастинация при общей отрицательной характеристике имеет некоторые адаптивные аспекты.

Позднее Corkin, Shirley, and Lindt [4,p.603], Klingsieck et al. [19, p.407] утверждали, что прокрастинация никаким образом не может сосуществовать с академической успешностью, а в обозначенных выше исследованиях авторы описывают скорее феномен функциональной или стратегический задержки, а не академической прокрастинации. Функциональная или стратегическая задержка предполагает, что субъект обладает развитой волевой регуляцией, он намеренно затягивает деятельность, сохраняя при этом и высокую мотивацию и уверенность в своих силах. Прокрастинирующие студенты не имели четких планов задержки работы, откладывая задачу, демонстрировали дистресс, сниженную самоэффективность и самооценку [21,p.48].

В исследовании, посвященном изучению причин и последствий академической прокрастинации, Grunschel, Patrzek и Fries [11,p.852] опросили 36 студентов и выявили внутренние и внешние причины академической прокрастинации. Внутренние причины включали восемь категорий: аффективные, психофизиологические, поведенческие характеристики, когнитивные установки в отношении личной компетентности, личностные особенности, ряд психологических особенностей, прошлый опыт обучения, воспринимаемые характеристики учебных задач. Внешние причины - три категории: индивидуальные условия труда, характеристики преподавателей, особенности самого учебного заведения. Чаще всего откладывались задачи, которые субъективно воспринимались студентами как сложные, вызывающие отвращение, важные, требующие больших временных затрат. К характеристикам преподавателя, способствующим прокрастинации студентов, были отнесены следующие: слишком расслабленный или слишком требовательный стиль преподавателя, неорганизованность, низкий уровень поддержки, низкий уровень компетентности, бескомпромиссность, неприятный внешний вид.

\section{Что делать в случае выявления академической прокрастинашии?}

Существующая на данный момент литература предлагает скудные примеры психологических программ, направленных на решение проблемы академической прокрастинации. При этом в большинстве из них не проводилась оценка эффективности вмешательств с помощью рандомизированных контролируемых исследований [31,р.1488]. Различные методы, которые приводятся в программах, можно рассматривать только как переложение теорий академической прокрастинацинаци, но не как результат системного подхода к интервенции.

Все психологические программы помощи в случаи академической прокрастинации можно условно разделить на три направления: психотерапия академической прокрастинации; психопрофилактика академической прокрастинации; программы помощи с участием педагогов. Первые два подхода схожи в том, что они используют психотерапевтические техники в работе с прокрастинацией. Различаются они тем, что программы первого направления начинают реализовываться после того, как студент продемонстрировал прокрастинаторное поведение, в то время как психологическая профилактика направлена на предотвращение негативных последствий прокрастинации, например, профилактические курсы могут предлагаться студентам в начале семестра [24,p.37]. Третий подход заключается в том, чтобы обучить преподавателя реализации непсихологических методов снижения прокрастинации среди студентов.

Масштабная оценка эффективности основных психологических методов снижения прокрастинации была предпринята Balkis \& Duru в 2007 году [2,p.380]. Авторы утверждают, что поведенческие методы терапии являются наиболее эффективными. Результаты были положительными как в терапевтической профилактике, так и в рамках коррекционных программ. Одним из примеров подобных исследований является оценка эффективности групповой программы, основанной на модели АВC А. Эллиса [23,p.125]. Программа состоит из пяти 90-минутных сессий, проводимых еженедельно. Она охватывает несколько блоков: изучение индивидуальных паттернов прокрастинации; когнитивная работа с иррациональными мыслями; поведенческая тренировка навыков продуктивной работы. Студенты, посещающие программу, показали значимое снижение академической и общей прокрастинации. Еще одно исследование [41,p.97] демонстрирует эффективную работу в условиях 
групповой терапии. Студентов-участников учили, как побороть прокрастинацию с помощью одной из двух программ. Первая программа была сфокусирована на модификации поведения с помощью управления внешними стимулами-предвестниками прокрастинации. Вторая программа была ориентирована на обучение студентов навыкам тайм-менеджмента. Участники обеих программ продемонстрировали как улучшение успеваемости, так и снижение прокрастинации.

В исследовании Scent \& Boes сообщается об эффективности групповой программы, основанной на принципах терапии принятия и ответственности. В рамках этой программы студентам помогали опознать прокрастинацию как форму избегающего поведения, расширить репертуар копинг-стратегий, осознать свои личные цели и ценности, укрепить учебную мотивацию. По окончании программы у молодых людей уменьшилась академическая прокрастинация, улучшилась учеба, повысилась психологическая гибкость - черта отражающая возможность человека выбирать наиболее эффективный для ситуации стиль поведения.

Какими бы успешными не были перечисленные программы, у них есть ряд существенных ограничений. Вопервых, для их реализации необходимо привлекать профессиональных психологов-консультантов, во-вторых, программы не адаптированы для российских студентов и специалистов, в-третьих, данные программы реализуются в качестве отдельного курса, который не заложен в учебные планы университетов, предполагают добровольное обращение студентов, а далеко не все хронические прокрастинаторы осознают откладывание как проблему.

По этим причинам более предпочтительными являются программы третьего направления, реализуемые самими преподавателями, прошедшими краткое обучение. Таких программ немного, но они достаточно эффективны и просты в осуществлении, не предполагают наличие специальных психологических знаний и навыков, прохождения долгосрочного обучения в рамках психотерапевтических школ. Они могут быть реализованы без отрыва студентов от основного обучения и не требуют сложных диагностических мероприятий, подходят как для помощи уже страдающим прокрастинацией студентам, так и для профилактики откладывания. Кратко остановимся на том, о каких методах идет речь. Прежде всего, это рекомендации по контролю знаний. Так небольшие еженедельные проверочные работы или викторины помогают студентам оставаться в тонусе и осваивать программу дисциплины, не откладывая подготовку на сессионный период [40,p.142]. В рамках дистанционного обучения предлагается делать контрольные работы условием доступа к прохождению следующей части курса, использовать модульную систему органи- зации курса, что позволяет не только снизить прокрастинацию, но и оценивать компетентность студентов на протяжении всего семестра, а также более эффективно составлять содержание итоговых испытаний по курсу [25,p.468]. Еще один инструмент предложили Strunk and Spencer [39,p.93]. В том случае, если студент не смог вовремя предоставить работы или плохо сдал сессию, в начале следующего семестра ему назначалась встреча с инструктором или тютором курса, на которой обсуждались последствия прокрастинации, инструктор помогал студенту составить письменный план закрытия учебных задолженностей, план подписывался студентом и инструктором. Инструктор следил за реализацией плана и оказывал помощь в планировании деятельности в течение семестра. При оценке эффективности этого приема оказалось, что вовлеченные в программу помощи студенты, действительно сдали прошлые задолженности, в новом семестре получали более высокие оценки и не накопили долгов.

Стоит упомянуть еще одну программу, в которой использовалась система СМС оповещений для напоминания студентам о ежедневных заданиях и еженедельной отчетности [5,p.87]. Данная система была разработана на основе принципов оперантного обусловливания. Если преподаватель не фиксировал в системе факт сдачи задания, то СМС-сообщения продолжали поступать студенту и превращались в негативные раздражители, от которых хотелось избавиться [3].

К сожалению, среди программ снижения академической прокрастинации нет таких, целью которых было бы изменение преподавательского стиля или изменение методики преподавания курса. Кроме того, все перечисленные выше программы требуют реорганизации учебного плана, дополнительного финансирования, что может являться препятствием для их реализации.

\section{Зак^ючение}

Обзор исследований академической прокрастинации дает возможность сделать некоторые выводы. Очевиден разрыв между распространённостью академической прокрастинации, обилием психологических исследований факторов ее развития и крайне скудным набором программ помощи [9,p.400]. Причин такого несоответствия может быть несколько: недостаточно четкое определение понятия академической прокрастинации; отсутствие доступных инструментов ее исследования, измерения и дифференциации от сходных явлений; разнообразие теоретических моделей, не позволяющее реализовать универсальную программу помощи; недооценка проблемы преподавателями и администрацией ВУЗов, а также самими студентами; сложности разработки и реализации программ помощи. 
При организации программ помощи важно понимать, что прокрастинация может иметь разные причины. Это может быть поведение в связи с личностной характеристикой студента, в этом случае требуется индивидуальная психологическая помощь. Если речь идет о прокрастинации как следствии несформированности навыков планирования деятельности, то помощь таким студентам может быть оказана самими преподавателями или тьюторами. Если прокрастинация в данном образовательном учреждении носит массовый характер и связана с организацией учебного процесса, со стилем преподавателей, в этом случае требуется работа адми- нистрации по устранению указанных причин.

Данная статья призвана привлечь внимание педагогов к проблеме прокрастинации у студентов и старших школьников, мотивировать их на то, чтобы самостоятельно разбираться с этим явлением и учитывать в рамках своей ежедневной работы. Воспитательная функция крайне важна в процессе работы преподавателей ВУЗа, педагоги несут профессиональную и личную ответственность за качество подготовки специалистов и они в силах помочь молодым людям учиться лучше, получать удовольствием от обучения и от будущей профессиональной деятельности.

\section{ЛИТЕРАТУРА}

1. Детство XXI века: социогуманитарный тезаурус: [тематический словарь-справочник] [Электронный ресурс] / Отв. ред. С.Н.Майорова-Щеглова. М.: ИздBO POC, 2018. $638 \mathrm{C}$

2. Balkis, M. (2011). Academic efficacy as a mediator and moderator variable in the relationship between academic procrastination and academic achievement. Egitim Arastirmalari Eurasian. Journal of Educational Research, 45, 1-16.

3. Balkis, M., \& Duru, E. (2007). The evaluation of the major characteristics and aspects of the procrastination in the framework of psychological counseling and guidance. Educational Sciences: Theory \& Practice, 7(1), 376-385.

4. Cooper, J.O., Heron, T.E., \& Heward, W.L. (2007). Applied Behavior Analysis. Upper Saddle River, NJ: Prentice Hall.

5. Corkin, D.M., Shirley, L.Y., \& Lindt, S.F. (2011). Comparing active delay and procrastination from a self-regulated learning perspective. Learning and Individual Differences, 21(5), 602-606. doi:10.1016/j.lindif.2011.07.005

6. Davis, D.R., \& Abbitt, J.T. (2013). An investigation of the impact of an intervention to reduce academic procrastination using short message service (SMS) technology. Journal of Interactive Online Learning, 12(3), 78-102.

7. Ferrari, J.R. (2001). Procrastination as self-regulation failure of performance: Effects of cognitive load, self-awareness, and time limits on 'working best under pressure.' European Journal of Personality, 15(5), 391-406. doi:10.1002/per.413.abs

8. Ferrari, J.R., Keane, S.M., Wolfe, R.N., \& Beck, B.L. (1998). The antecedents and consequences of academic excuse-making: Examining individual differences in procrastination. Research in Higher Education, 39(2), 199-215.

9. Flett, G.L., Stainton, M., Hewitt, P.L., Sherry, S.B., \& Lay, C. (2012). Procrastination automatic thoughts as a personality construct: An analysis of the procrastinatory cognitions inventory. Journal of Rational-Emotive \& Cognitive-Behavior Therapy, 30(4), 223-236. doi:10.1007/s10942-012-0150-z

10. Glick, D.M., \& Orsillo, S.M. (2015). An investigation of the efficacy of acceptance-based behavioral therapy for academic procrastination. Journal of Experimental Psychology: General, 144(2), 400. doi:10.1037/xge0000050

11. Gropel, P., \& Steel, P. (2008). A mega-trial investigation of goal setting, interest enhancement, and energy on procrastination. Personality and Individual Differences, 45(5), 406-411. doi:10.1016/j.paid.2008.05.015

12. Grunschel, C., Patrzek, J., \& Fries, S. (2013). Exploring reasons and consequences of academic procrastination: An interview study. European Journal of Psychology of Education, 28(3), 841-861. doi:10.1007/s10212-012-0143-4

13. Gustavson, D.E., Miyake, A., Hewitt, J.K., \& Friedman, N.P. (2014). Genetic relations among procrastination, impulsivity, and goal-management ability implications for the Haghbin, M., McCaffrey, A., \& Pychyl, T.A. (2012). The complexity of the relation between fear of failure and procrastination. Journal of Rational-Emotive \& Cognitive-Behavior Therapy, 30(4), 249-263. doi:10.1007/s10942-012-0153-9

14. Hen, M., \& Goroshit, M. (2014). Academic procrastination, emotional intelligence, academic self-efficacy, and GPA: A Comparison between students with and without learning disabilities. Journal of Learning Disabilities, 47(2), 116-124. doi:10.1177/0022219412439325

15. Kim, K.R., \& Seo, E.H. (2015). The relationship between procrastination and academic performance: A meta-analysis. Personality and Individual Differences, 82 , 26-33. doi:10.1016/j. paid.2015.02.038

16. Klassen, R.M., Krawchuk, L.L., \& Rajani, S. (2008). Academic procrastination of undergraduates: Low self-efficacy to self-regulate predicts higher levels of procrastination. Contemporary Educational Psychology, 33(4), 915-931. doi:10.1016/j.cedpsych.2007.07.001

17. Klingsieck, K.B. (2013a). Procrastination in different life-domains: Is procrastination domain specific? Current Psychology, 32(2), 175-185. doi:10.1007/s12144013-9171-8

18. Klingsieck, K.B. (2013b). Procrastination: When good things don't come to those who wait. European Psychologist, 18(1), 24-34. doi:10.1027/1016-9040/a000138

19. Klingsieck, K.B., Grund, A., Schmid, S., \& Fries, S. (2013). Why students procrastinate: A qualitative approach. Journal of College Student Development, 54(4), 397-412. doi:10.1353/ csd.2013.0060

20. Krause, K., \& Fruend, A.M. (2014). Delay or procrastination - A comparison of self-report and behavioral measures of procrastination and their impact on affective 
well-being. Personality and Individual Differences, 63, 75-80. doi:10.1016/j.paid.2014.01.050

21. Lindblom-Ylänne, S., Saariaho, E., Inkinen, M., Haarala-Muhonen, A., \& Hailikari, T. (2015). Academic procrastinators, strategic delayers and something betwixt and between: An interview study. Frontline Learning Research, 3(2), 47-62.

22. Onwuegbuzie, A.J., \& Jiao, Q.G. (2000). I'll go to the library later: The relationship between academic procrastination and library anxiety. College \& Research Libraries, 61(1), 45-54. doi:10.5860/crl.61.1.45

23. Ozer, B.U., Demir, A., \& Ferrari, J. R. (2013). Reducing academic procrastination through a group treatment program: A pilot study. Journal of Rational-Emotive \& Cognitive-Behavior Therapy, 31(3), 127-135. doi:10.1007/s10942-013-0165-0

24. Ozer, B.U., \& Ferrari, J.R. (2011). Gender orientation and academic procrastination: Exploring Turkish high school students. Individual Differences Research, 9(1), $33-40$.

25. Perrin, C.J., Miller, N., Haberlin, A.T., Ivy, J.W., Meindl, J.N., \& Neef, N.A. (2011). Measuring and reducing college students' procrastination. Journal of Applied Behavior Analysis, 44(3), 463-474. doi:10.1901/jaba.2011.44-463

26. Pintrich, P.R. (2004). A conceptual framework for assessing motivation and self-regulated learning in college students. Educational Psychology Review, 16(4), 385-407. doi:10.1007/s10648-004-0006- $x$

27. Pychyl, T.A., \& Flett, G.L. (2012). Procrastination and self-regulatory failure: An introduction to the special issue. Journal of Rational-Emotive \& Cognitive-Behavior Therapy, 30(4), 203-212. doi:10.1007/s10942-012-0149-5

28. Rabin, L.A., Fogel, J., \& Nutter-Upham, K.E. (2011). Academic procrastination in college students: The role of self-reported executive function. Journal of Clinical and Experimental Neuropsychology, 33(3), 344-357. doi:10.1080/13803395.2010.518597

29. Rebetez, M.M.L., Rochat, L., \& Van der Linden, M. (2015). Cognitive, emotional, and motivational factors related to procrastination: A cluster analytic approach. Personality and Individual Differences, 76, 1-6. doi:10.1016/j.paid.2014.11.044

30. Rice, K.G., Richardson, C.M., \& Clark, D. (2012). Perfectionism, procrastination, and psychological distress. Journal of Counseling Psychology, $59(2), 288$. doi:10.1037/a0026643

31. Rozental, A., \& Carlbring, P. (2014). Understanding and treating procrastination: A review of a common self-regulatory failure. Psychology, $5(13), 1488$. doi:10.4236/psych.2014. 513160

32. Scent, C.L., \& Boes, S.R. (2014). Acceptance and commitment training: A brief intervention to reduce procrastination among college students. Journal of College Student Psychotherapy, 28(2), 144-156. doi:10.1080/87568225.2014.883887

33. Schraw, G., Wadkins, T., \& Olafson, L. (2007). Doing the things we do: A grounded theory of academic procrastination. Journal of Educational Psychology, $99(1), 12$. doi:10.1037/0022-0663.99.1.12

34. Sirois, F.M., \& Tosti, N. (2012). Lost in the moment? An investigation of procrastination, mindfulness, and well-being. Journal of Rational-Emotive \& CognitiveBehavior Therapy, 30(4), 237-248. doi:10.1007/s10942-012-0151-y

35. Steel, P. (2007). The nature of procrastination: A meta-analytic and theoretical review of quintessential self-regulatory failure. Psychological Bulletin, $133(1), 65$. doi:10.1037/0033-2909.133.1.65

36. Steel, P. (2010). The procrastination equation: How to stop putting things off and start getting stuff done. Toronto: Random House Canada.

37. Steel, P., \& Ferrari, J. (2013). Sex, education and procrastination: An epidemiological study of procrastinators' characteristics from a global sample. European Journal of Personality, 27(1), 51-58. doi:10.1002/per.1851

38. Steel, P., \& Klingsieck, K.B. (2016). Academic procrastination: Psychological antecedents revisited. Australian Psychologist, 51(1), 36-46. doi:10.1111/ap.12173 JOURNAL OF PREVENTION \& INTERVENTION IN THE COMMUNITY129 evolutionary origin of procrastination. Psychological Science, 25(6), 1178-1188. doi:10.1177/ 0956797614526260

39. Strunk, K.K., \& Spencer, J.M. (2012). A brief intervention for reducing procrastination. Academic Exchange Quarterly, 16(1), 91-96.

40. Tuckman, B.W. (1998). Using tests as an incentive to motivate procrastinators to study. The Journal of Experimental Education, 66(2), 141-147. doi:10.1080/00220979809601400

41. Tuckman, B.W., \& Schouwenburg, H.C. (2004). Behavioral interventions for reducing procrastination among university students. In H. C. Schouwenburg, C.H. Lay, T.A. Pychyl, \& J.R. Ferrari (Eds.), Counseling the procrastinator in academic settings (pp. 91-103). Washington, DC: American Psychological Association.

42. Van Eerde, W. (2003). A meta-analytically derived nomological network of procrastination. Personality and Individual Differences, 35(6), 1401-1418. doi:10.1016/ s0191-8869(02) 00358-6

43. Wäschle, K., Allgaier, A., Lachner, A., Fink, S., \& Nückles, M. (2014). Procrastination and self-efficacy: Tracing vicious and virtuous circles in self-regulated learning. Learning and Instruction, 29, 103-114. doi:10.1016/j.learninstruc.2013.09.005

44. Zimmerman, B.J. (2002). Becoming a self-regulated learner: An overview. Theory into Practice, 41(2), 64-70. doi:10.1207/s15430421tip4102_2

(c) Коновалова Анастасия Вадимовна (konanavad@gmail.com).

Журнал «Современная наука: актуальные проблемы теории и практики» 